Elsevier

HEARES 01245

\title{
The organization of tip links and stereocilia on hair cells of bird and lizard basilar papillae
}

\author{
J.O. Pickles ${ }^{1,2}$, J. Brix ${ }^{3}$, S.D. Comis ${ }^{2}$, O. Gleich ${ }^{3, *}$, C. Köppl ${ }^{3}$, G.A. Manley ${ }^{3}$ \\ and M.P. Osborne ${ }^{2}$ \\ 'Vision Touch and Hearing Research Centre, University of Queensland, St. Lucia, Queensland, Australia, ${ }^{2}$ Department of Physiology, \\ University of Birmingham, Birmingham, U.K. and ${ }^{3}$ Institut für Zoologie, Technische Universität München, Garching, F.R.G.
}

(Received 12 January 1988; accepted 16 April 1989)

\begin{abstract}
Auditory papillae from three species of bird (pigeon, starling, and chick), and two species of European lizard (Podarcis muralis and Podarcis sicula) were examined by scanning electron microscopy. Hair bundles from all papillae showed tip links oriented along the direction of gradation in heights of the stereocilia (i.e. parallel to the hair-cell axis of bilateral symmetry, and so parallel to the excitatory-inhibitory axis for mechanotransduction). This orientation was seen irrespective of the overall orientation of the hair bundle within the papilla. The stereocilia formed columns, joined by the tip links, which ran parallel to the hair-cell axis of bilateral symmetry. The stereocilia within the same column tended to stay together, while those in different columns tended to separate during preparation. In many columns all the stereocilia tended to be a little taller, or a little shorter, than the equivalent stereocilia in adjacent columns, suggesting that all the stereocilia within one column had been affected by a common height determinant during development. In addition, links running laterally between stereocilia were seen, in a band near the base of the stereocilia. The results are consistent with the hypothesis that tip links are a universal feature of mechano-transducing acousticolateral hair cells, and that they are involved in sensory transduction. The results also support suggestions that the tip links may play a role in determining the heights of the stereocilia during development.
\end{abstract}

Key words: stereocilia; hair cell; bird; lizard; papilla; link; tip link; transduction; development.

\section{Introduction}

The linkages between stereocilia (stereovilli) have previously been described extensively in mammals. The links can be divided into two broad groups (Osborne et al., 1984; Pickles et al., 1984). Links of one group join the stereocilia laterally, and run predominantly parallel to the cuticular plate. These may well have a mechanical function, so that forces transmitted to some stereocilia on a

Correspondence to: Dr. J.O. Pickles, Vision, Touch and Hearing Research Centre, Department of Physiology and Pharmacology, University of Queensland, St. Lucia, Queensland 4067, Australia.

* Present address: Department of Physiology, University of Western Australia, Nedlands, W.A. 6009, Australia. hair cell bundle are transmitted to other stereocilia in the bundle (Flock and Strelioff, 1984). Links in the second group emerge from the tips of each of the shorter stereocilia in a bundle, and run at a steep angle to the cuticular plate, to join the side-wall of the adjacent taller stereocilium in the bundle. It has been suggested that links of this second group, known as tip links, are involved in mechano-transduction. They might be stretched by a stimulus-induced shear between the different rows of stereocilia, and so open transducer channels situated at one or other of their points of attachment to the stereocilia (Pickles et al., 1984; Pickles, 1985; Hudspeth, 1985).

Tip links have been previously described in several species of mammal, including man (Osborne et al., 1984; Pickles et al., 1984; RhysEvans et al., 1985; Lim, 1986; Lenoir et al., 1987), 
and also in hair cells of the fish saccular macula (Little and Neugebauer, 1985). However, there are no clear descriptions published for other species, such as birds and reptiles. If tip links are involved in transduction, it is important to see whether they exist in those species as well. Moreover, it may be possible to gain additional information about their function by investigating a wider range of species.

Observations in the guinea-pig organ of Corti indicate that the tip links have a horizontal component in their orientation which runs parallel to the axis of bilateral symmetry of the hair bundle (i.e. parallel to the line drawn between the kinocilium or its basal body and the centre of the hair bundle; Pickles et al., 1984; Comis et al., 1985). This axis also defines the direction for maximum sensitivity to deflection of the stereocilia during mechano-transduction (Lowenstein and Wersäll, 1959; Flock, 1965; Hudspeth and Corey 1977). That relation forms important corroborative evidence for the hypothesis that tip links might be involved in mechano-transduction.

In the present study, the spatial organization of tip links has been analyzed in bird and lizard hair bundles. In comparison with the guinea pig cochlea, the bundles have a compact conformation, so the orientation of the tip links can be studied more reliably. Moreover, in reptiles and birds the hair bundles may have a variety of orientations with respect to the neural edge of the organ, with particularly interesting implications for the orientation of the tip links. In lizards for instance some groups of hair cells may be bidirectionally oriented (Miller, 1978; Manley, 1989), and in the birds the orientation of the hair bundles changes in a regular and systematic manner across the width of the papilla (Tilney et al., 1987; Gleich and Manley, 1988; Fischer et al., 1988).

We show in these bundles, in spite of their very different conformations and orientations, that with very few exceptions the tip links run parallel to the hair cell's axis of bilateral symmetry. Moreover, we describe a new factor in the organization of stereocilia, namely the presence of columns of stereocilia joined by tip links, with the columns running parallel to the axis of bilateral symmetry.

Preliminary reports of some aspects of the present results have been given previously (Pickles et al., 1988a, b).

\section{Methods}

The study was performed on three pigeons, Columba livia, two starlings, Sturnus vulgaris, six chicks, Gallus gallus domesticus, two European wall lizards, Podarcis muralis and one European ruin lizard, Podarcis sicula. The subjects were anaesthetized and the inner ear perfused with fixative, either by transcardial infusion or direct introduction of the fixative into the inner ear. In pigeons the fixative was $2.5 \%$ glutaraldehyde in $0.1 \mathrm{M}$ phosphate buffer. In chicks, starlings and lizards the fixative was $1 \%$ glutaraldehyde and $15 \%$ saturated picric acid in $0.1 \mathrm{M}$ phosphate buffer. Specimens were stored in $2 \%$ glutaraldehyde in $0.05 \mathrm{M}$ phosphate buffer until further treatment. They were then dehydrated in acetone, dried by the critical-point technique with liquid $\mathrm{CO}_{2}$, and sputter-coated with platinum to a nominal depth of $25 \mathrm{~nm}$. Specimens were examined at $40 \mathrm{kV}$ in a JEOL $120 \mathrm{CXII}$ transmission electron microscope with a scanning attachment (TEMSCAN), and images were observed by a secondaryelectron detector.

\section{Results}

Shapes of bundle, packing of stereocilia, and directions of tip links

At the apical (distal, or low-frequency) end of the birds' basilar papillae the hair bundles were tall and narrow (e.g. for the starling, Fig. 1; Gleich and Manley, 1988). Sometimes, as here, the stereocilia on the lateral edge of the bundle bowed outwards in the middle, while remaining attached at their upper ends. Tip links were visible emerging from the shorter stereocilia in the bundle (Fig. 2 ), and in undistorted bundles ran in the direction of the gradient in the heights of the stereocilia, i.e. parallel to the hair cell's axis of bilateral symmetry. A similar conformation for apical hair cells was seen in pigeons and chicks.

At the middle and basal ends of the bird papillae the bundles were wider, and contained more stereocilia. The shape of the bundle varied with the hair cell's position both along the apical-basal axis of the papilla, and along the neural-abneural (i.e. superior-inferior) axis, although the exact relations were not quantified in the present study 

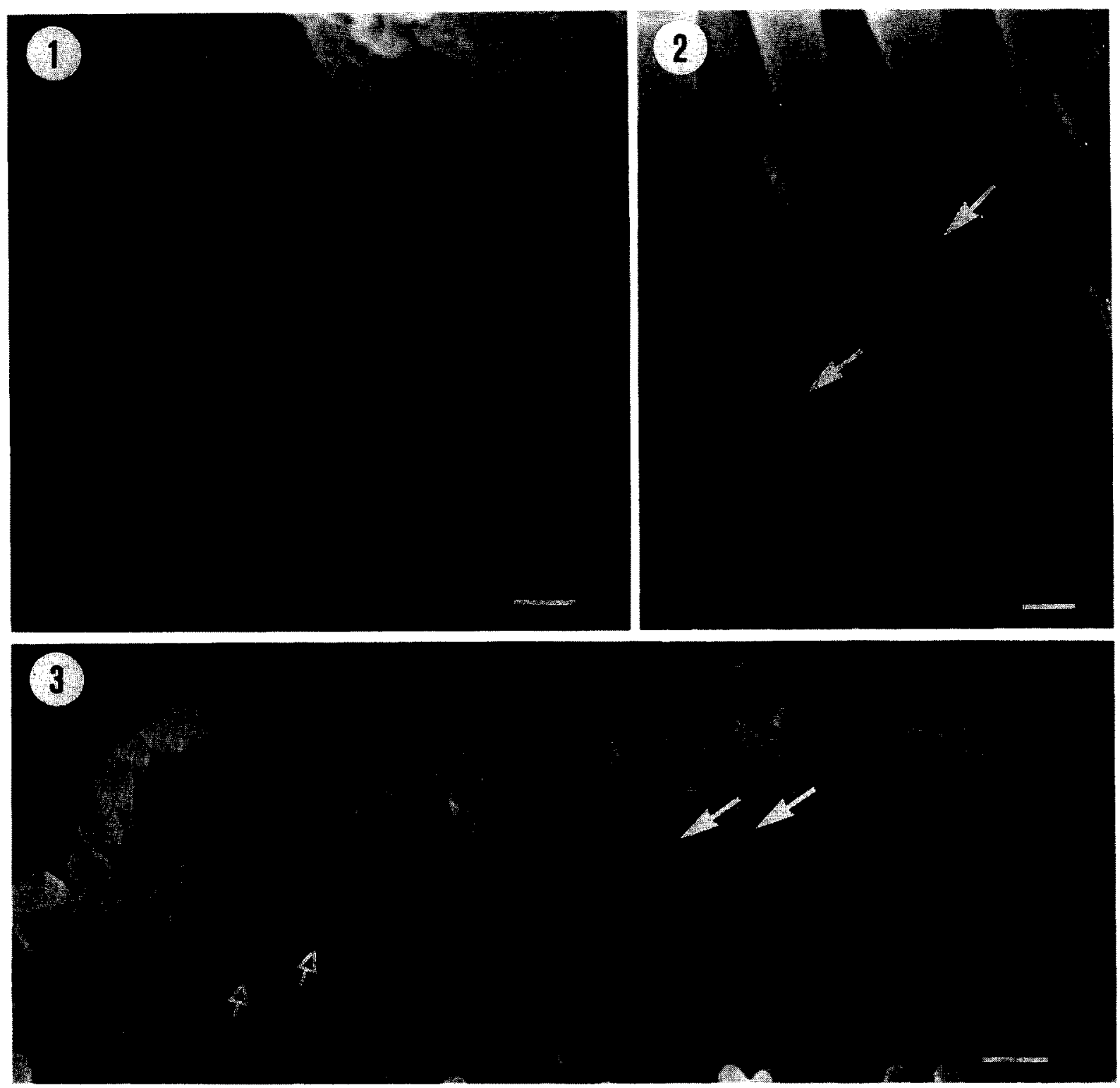

Fig. 1. Hair cell of the apical region of the starling basilar papilla. The material at the top right represents debris, possibly from the tectorial membrane. Debris is also attached to the tops of the tallest stereocilia. Scale bar $=500 \mathrm{~nm}$.

Fig. 2. Detail of the hair cell in Fig. 1. Arrows point to some of the visible tip links. Scale bar $=200 \mathrm{~nm}$.

Fig. 3. Hair cell of the starling basilar papilla, situated on the neural edge of the papilla, and midway between its apical and basal ends. Closed arrows point to some of the visible tip links. Open arrows show columns of stereocilia in which all the stereocilia are a little taller than those in adjacent columns. Debris is attached to the tops of the tallest stereocilia. Composite of three micrographs of same hair bundle. Scale bar $=500 \mathrm{~nm}$.

(see however Tilney et al., 1987; Gleich and Manley, 1988; Fischer et al., 1988; Manley, 1989). A hair cell for the starling is shown in Fig. 3, and for the chick in Fig. 4. Tip links were visible emerging from the apices of the shorter stereocilia. When viewed in a direction perpendicular to the cuticu- 

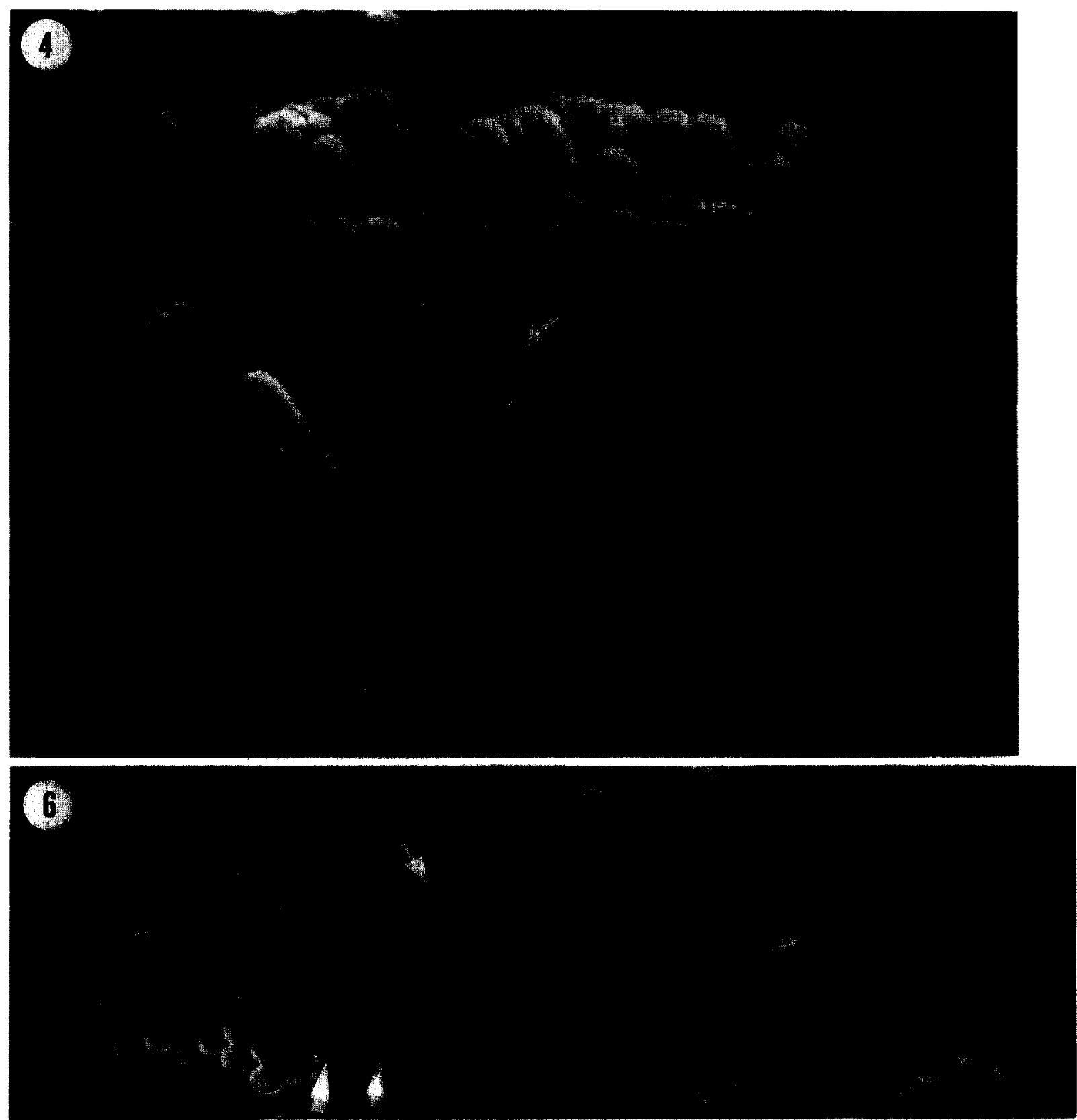

Fig. 4. Hair cell of the chick basilar papilla, situated medially along the papilla. Two of the columns have separated (arrowhead). Arrows point to some of the visible tip links. Double arrowhead: basal links. Scale bar $=500 \mathrm{~nm}$.

Fig. 6. Hair call of starling basilar papilla (midway apical-basal, on neural edge), viewed nearly perpendicular to the cuticular plate. Arrows: tip links. At the single arrowhead, the stereocilia have been separated, presumably as a result of a pull applied by the tectorial membrane contacting the tallest stereocilia (towards top of picture). In the rest of the bundle the stereocilia remain hexagonally packed, with tip links joining stereocilia in col'mns along the $(1, \overline{1})$ plane (double arrowheads). Debris overlies the tallest stereocilia. Composite of three micrographs of same hair bundle. Scale bar $=500 \mathrm{~nm}$. 


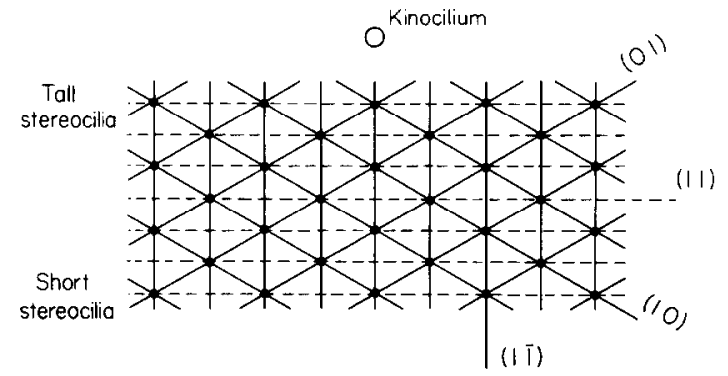

Fig. 5. The nomenclature of planes (Miller indices) in a hexagonally-packed structure, consistent with the nomenclature of Tilney et al. (1986).

lar plate, the stereocilia were seen to be hexagonally packed (Fig. 6). The tip links ran along a $(1, \overline{1})$ plane of the hexagon (defined in Fig. 5) and almost at right angles to the long axis of the bundle, so that the tip links ran parallel to the hair cell's axis of bilateral symmetry (Fig. 6).

In European lizards the basilar papilla is divided into two separate subpapillae. A basic description of papillar morphology can be found in Wever (1978) and Miller (1978). A more extensive description is in preparation by one of us (C. Köppl). Fig. 7 shows a general view of the basal subpapilla of the lizard Podarcis sicula. The overlying material has been removed from the hair cells on the basal portion of the basal subpapilla (centre and left of figure). In this region, the hair cells are situated in two rows, of opposite polarity, one on either side of the papilla. The bundles were compact, approximately as deep as they were wide, with hexagonally packed stereocilia. The stereocilia were joined by tip links, running in the direction of the gradation in heights of the stereocilia. As in the birds, the direction of the links corresponded to the $(1, \overline{1})$ plane of the hexagonal packing of the stereocilia (Fig. 8). Similar observations were made from the basal subpapilla of the lizard Podarcis muralis (Fig. 9). In both species it was not easy to see the organization in the hair cells in the apical part of the basal subpapilla, nor in the apical subpapilla, because the tectorial membrane always remained partially attached. However, where stereocilia and their tip links were visible, the same organization was evident in both these regions.

\section{Columnar organization of the stereocilia}

Within the hexagonal packing of the stereocilia, a further organization was visible. The tip links defined columns, running along a $(1, \overline{1})$ plane of the hexagon in the direction of gradation of heights of the stereocilia, and running parallel to the hair cell's axis of bilateral symmetry. Figs. 3, 4, 6, 8, 9 and 11 show such columns, one of the most obvious examples being shown in Fig. 10. In the avian papilla, the columns were more readily apparent in the bundles in the middle and basal areas of the papilla than in the tall and narrow apical bundles, perhaps because the latter bundles more readily suffered small degrees of distortion during preparation. The tip links always ran along the direction of the column. In many cases the stereocilia in adjacent columns had separated slightly, cmphasising the columnar arrangement (e.g. Figs. 3 and 8 ). In other cases, the stereocilia of the different columns had separated widely, perhaps due to trauma during preparation of the specimen, while the stereocilia within the same column tended to stay together (Fig. 4).

In the middle of the bundles the columns usually continued uninterruptedly from the shorter stereocilia to the taller (e.g. Fig. 3). However, particularly near the edge of the bundle, it was common for two adjacent columns to join into a single column, so that the side-wall of a single taller stereocilium received the upper ends of the tip links emerging from the tips of two shorter stereocilia. Examples, typical for all species, are shown in Figs. 8 and 9. By contrast, the reverse condition, of one shorter stereocilium giving rise to two tip links, leading to the branching of a column as one moves in the direction of the taller stereocilia, was never unambiguously seen.

In birds, the orientation of the bundles changes in a systematic and regular way across the width of the papilla (Tilney et al., 1987; Gleich and Manley, 1988; Fischer et al., 1988). Nevertheless, in all bundles the tip links continued to run parallel to the hair cell axis of bilateral symmetry, irrespective of the hair cell's orientation within the papilla. In addition, within the systematic and regular change in hair cell orientation across the papilla, it was sometimes possible to see occasional cells with an orientation quite different from their neighbours. Fig. 11 shows an example 

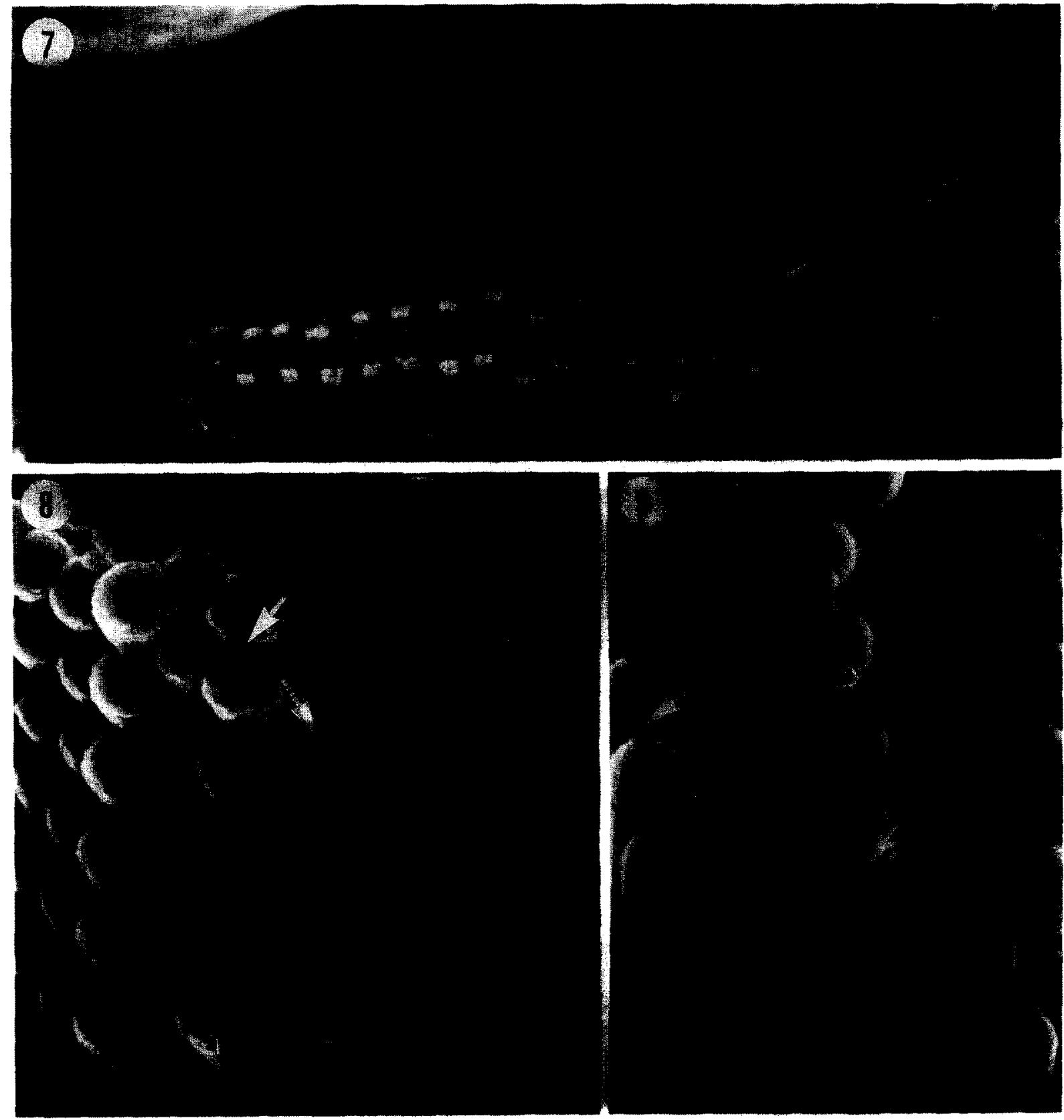

Fig. 7. The basal subpapilla of the ruin lizard Podarcis sicula. The tectorial plate (right) has shrunk, and pulled away from the underlying hair cells, the bundles of which are disorganized. The material over the hair cells in basal portion of the basal subpapilla (centre and left) has been removed. Arrow: hair cell shown in Fig. 8 . Scale bar $=10 \mu \mathrm{m}$.

Fig. 8 Hair cell of the basal portion of the basal subpapilla of the ruin lizard Podarcis sicula, showing tip links (e.g. arrows) and columns of stereocilia (open arrows). At the arrowheads two columns join. Scale bar $=200 \mathrm{~nm}$.

Fig. 9. Hair cell of the basal portion of the basal subpapilla of the wall lizard Podarcis muralis, showing tip links (e.g. arrows) and columns of stereocilia (open arrows). At the arrowhead two columns join. Scale bar $=200 \mathrm{~nm}$. 

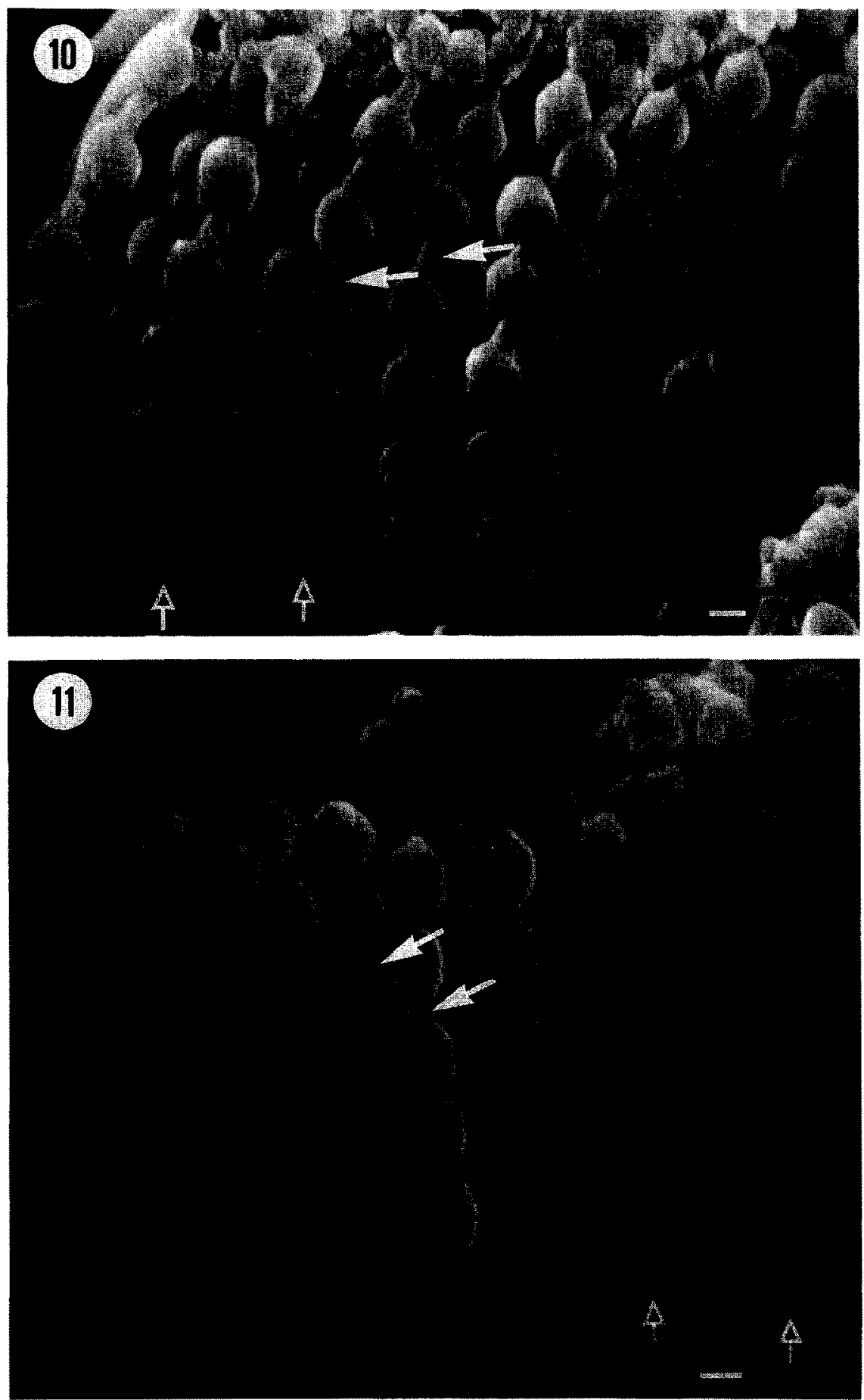

Fig. 10. Columns of stereocilia joined by tip links (e.g. closed arrows) in a hair cell of the starling basilar papilla (midway apical-basal, on neural edge). Open arrows: columns in which stereocilia are a little taller than their neighbours. Some debris is attached to the tallest stereocilia. Scale bar $=200 \mathrm{~nm}$.

Fig. 11. Hair cell with abnormal orientation. The columns defined by the tip links run apparently at right angles to the long axis of the bundle. Closed arrows: examples of tip links. Open arrows: columns in which all the stereocilia are a little taller than in adjacent columns. Starling. Scale bar $=200 \mathrm{~nm}$. 
of a hair cell from a starling papilla, where the cell was oriented approximately $45-60^{\circ}$ differently from its immediate neighbours, as judged by the direction of the long axis of the bundle. However, the tip links and the columns of stereocilia in these rotated cells had the same orientation with respect to the bundle's own long axis (Fig. 11) as in all other hair cells.

\section{Variation in heights of the stereocilia}

It was commonly found that, within the general gradation in height of the stereocilia from shorter to taller within each column, all the stereocilia within one column could be a little taller, or a little shorter, than the corresponding stereocilia in adjacent columns. The open arrows in Figs. 3, 10, and 11 show examples of columns in which all the stereocilia tended to be taller than the corresponding stereocilia in adjacent columns. Such examples were seen in all species, and in hair cells from all parts of the papillae. In many cases no regularity was apparent in the positioning of the columns of taller or shorter stereocilia. For instance, there was no indication that columns of taller and shorter stereocilia were necessarily placed alternately. In view of these height variations, lines joining stereocilia of the same height in a bundle do not therefore always run exactly along the $(1,1)$ plane of the hexagon, defined in Fig. 5, as previously suggested by Tilney et al. (1986). Moreover, the most regular gradation in heights of the stereocilia is produced along the tip link column, i.e. the $(1, \overline{1})$ plane, rather than the $(1,0)$ and $(0,1)$ planes (a point also made by Tilney et al., 1988a).

\section{'Loose' and 'tight' bundles}

Bagger-Sjöbäck and Takumida (1988) divided bundles of the guinea-pig vestibular system into 'loose' and 'tight' bundles. In the more common loose bundles, lines drawn between stereocilia of equal height run along, or approximately along, the $(1,1)$ plane of the hexagon. These lines run at right angles to the $(1, \overline{1})$ plane containing the tip links. In the less common tight type, lines drawn between stereocilia of equal height may run along the $(1,0)$ or $(0,1)$ plane of the hexagon, these being oriented at $60^{\circ}$ to the $(1, \overline{1})$ plane containing the tip links. If one ignores the small height variations described in the previous section, it is possible to make a similar division among the bundles studied

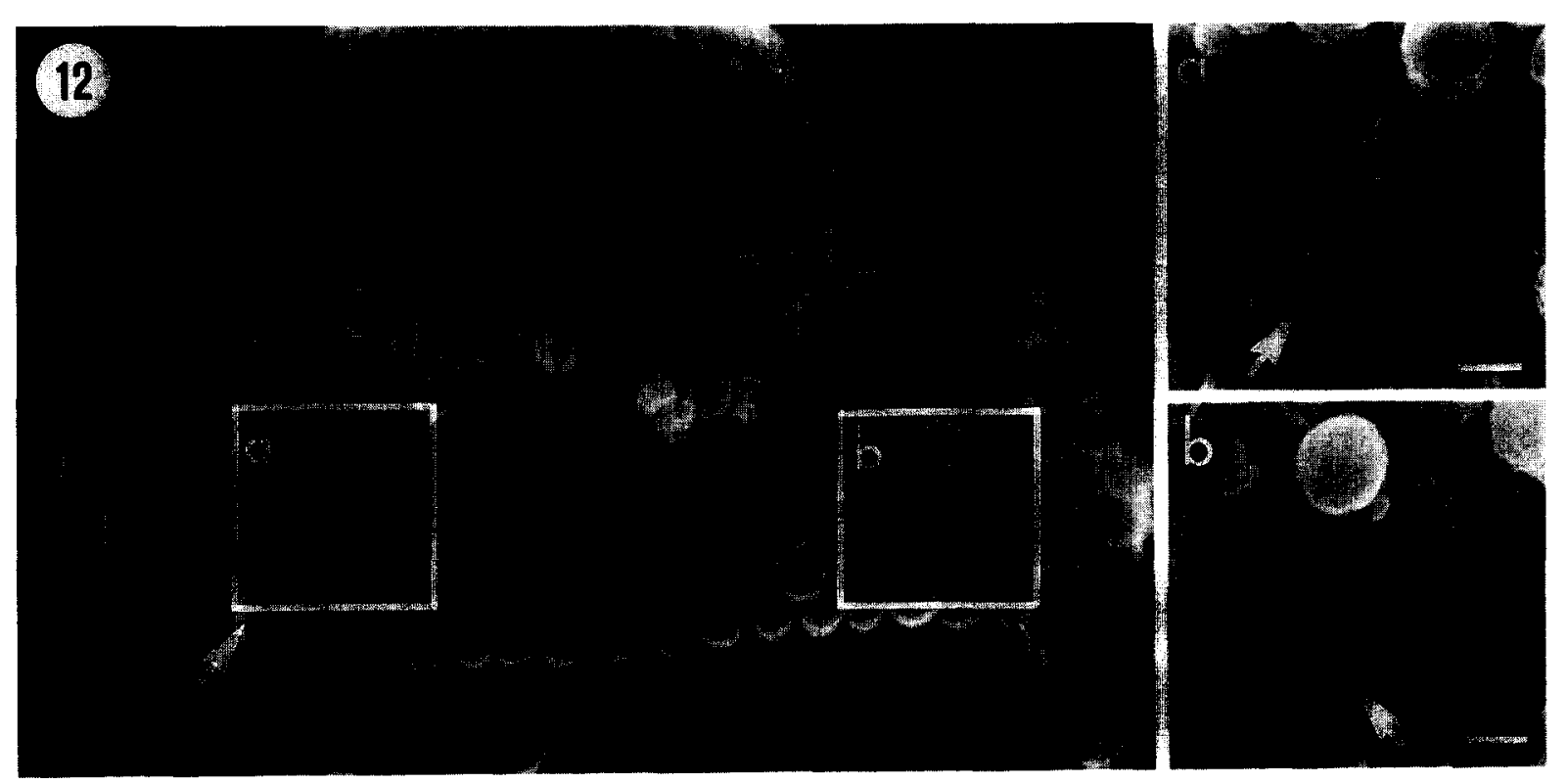

Fig. 12. Anomalous pigeon hair cell with columns (arrows) running in different directions. Inserts a and b: the areas shown in the corresponding white squares on main figure. The large swelling at the top of the figure is probably a preparation artefact, perhaps due to osmotic effects during fixation, and arising from the opening in the cuticular plate. (Not all anomalous cells had a bleb, nor were all cells with a bleb anomalous). Scale bar $=500 \mathrm{~nm}$ on main figure, $200 \mathrm{~nm}$ on insert. 
here. The very great majority of bundles analyzed, from both apical and basal parts of the papillae, were of the loose type. It was only in the apical quarter of the bird papillae that some bundles of the tight type were visible, and in most specimens they formed the minority.

\section{Lateral links between stereocilia}

As well as tip links, linkages joining the stereocilia laterally were visible. In all species studied in the present paper, the lateral links were visible in a band near the base of the stereocilia (e.g. Fig. 4). Where the stereocilia separated into columns, no obvious debris was visible on the upper parts of the lateral, separated, walls of the stereocilia of the different columns (Fig. 4). However in such cases, a close look deep into the gap could show debris on the lower lateral surfaces of the stereocilia, consistent with the remains of lateral linkages running between the columns near the base of the stereocilia.

\section{Bundles with anomalous organization}

In some birds, bundles were found which clearly did not fit in with the above scheme. One pigeon showed particularly dramatic differences, in the hair cells nearest to the neural (i.e. superior) edge of the papilla. Here, the bundles were commonly divided into two or more groups, with the columns defined by the tip links running in different directions in the two groups (Fig. 12). It should be emphasised that hair cells with anomalies as shown in Fig. 12 formed a very small proportion indeed of the total number of hair cells examined (less than $1 \%$ ).

\section{Discussion}

The present results extend to five additional species the observations, made initially in the guinea pig organ of Corti, that tip links exist in acousticolateral hair bundles. Tip links have now been found on hair cells from representatives of all vertebrate classes. It is therefore quite likely that they are universally present in acousticolateral mechanoreceptor hair cells. Now that tip links have been identified, the links or fragments of the links can be seen in earlier micrographs produced for other systems and species, although the authors did not point them out. For instance they can also be seen in the skink Mabuya carinata (Fig. 15 of Miller, 1978), and in the lateral line organ of the burbot Lota vulgaris (Fig. 10 of Flock, 1965).

The observations also extend to five additional species the finding that tip links have a horizontal component in their orientation which runs parallel to the hair cell's axis of bilateral symmetry (Pickles et al., 1984; Comis et al., 1985). The same point has been confirmed for hair cells of the fish saccular macula (Little and Neugebauer, 1985). The hair cells investigated in the present paper have an advantage over those in the guinea pig organ of Corti, in that the compact hexagonally-packed bundles are less vulnerable to mechanical disruption during preparation. Morcover, the greater number of rows of stereocilia mean that the directional organization of the tip links is more easily demonstrated.

The hair cells of the present study have also demonstrated a new principle in the spatial organization of hair cell stereocilia, namely, the organization of stereocilia into columns, joined by tip links, running parallel to the hair cell's axis of bilateral symmetry. Columns can also be seen in inner hair cells of the guinea pig organ of Corti (Comis et al., 1985; Fig. 3.4B in Pickles, 1988). However, they are more difficult to demonstrate in the latter hair cells, because the shape of the inner hair cell bundles means that the stereocilia easily suffer small distortions during preparation.

The orientation of tip links along a column is in agreement with the idea that the tip links are involved in mechano-transduction, since the axis of bilateral symmetry of the cell also defines the cell's functional excitatory-inhibitory axis in response to deflection of the stereocilia (Lowenstein and Wersäll, 1959; Flock, 1965; Hudspeth and Corey, 1977).

Because bundles during preparation tend to separate into columns defined by the tip links, it appears that the mechanical strength of linkages between columns is less than that of the linkages within a column. This is consistent with the results of Csukas et al. (1987), who found that in the alligator lizard there are no sideways links between the stereocilia in the upper part of the bundle. Assuming that alligator lizards have tip 
links, as do the European lizards investigated in the present study, this would leave the tip links as being the only connectors between stereocilia in the upper part of the bundle. The band of links joining the stereocilia sideways near the base, and previously described in a variety of species by Bagger-Sjöbäck and Wersäll (1973), Csukas et al. (1987) and Jeffries et al. (1987), were also visible in the present study, and in all the species investigated. They appeared to run between the stereocilia of the different tip-link columns near to their insertion into the cuticular plate, since where the columns had parted, it was sometimes possible to see the links or remains of links in the lower part of the gap.

One of the most intriguing questions underlying the present study is how the hair cell's axis of bilateral symmetry, the gradation in heights of the stereocilia, and the direction of the tip links, come to be so closely related. One possible hint comes from some observations made in this paper. It was noted that, as well as the overall gradation in heights of stereocilia from tall to short within a column, there could be small variations in height between columns. All the stereocilia in one column could he a little taller, and all those in another a little shorter, than the equivalent stereocilia in the adjacent columns (e.g. open arrows, Figs. 3, 10 and 11). It would therefore appear that a common factor can, within the normal gradation in heights of the stereocilia, influence the heights of all the stereocilia within the same tip-link column. This suggests that a factor has been transmitted along a column, as defined by the tip links, to govern the heights of the stereocilia during development. The observations support the hypothesis suggested by Tilney et al (1988b), who suggested that a pull on the tip links might induce growth of the shorter stereocilia. The pull on the links could open transducer channels, admitting ions such as $\mathrm{Ca}^{2+}$. The ions might then promote the growth of the shorter stereocilia by inhibiting the actin-capping proteins situated at their upper ends. In this way, a signal for stereociliar growth would be progressively transmitted from taller to shorter stereocilia along each individual tip-link column.

We are clearly far from understanding the factors which govern the organization of stereocilia and the tip links on the hair cell surface. Hypotheses would have to explain how tip links come to run parallel to the hair cell axis of bilateral symmetry. The hypotheses would also have to explain the way that columns of tip links sometimes join together near the edge of the bundle (e.g. Figs. 8 and 9), and why anomalous bundles as in Fig. 12 are so rare. Further problems are how the various patterns of hair cell orientation across the papilla develop, and why when some hair cells have an orientation different from their neighbours, the tip-link columns still appear to run parallel to the hair cell axis of bilateral symmetry (Fig. 11). Observations such as these, and those on the reversed guinea-pig hair cells described by Comis et al. (1989), form some of the data against which theories of hair-cell development can be more critically evaluated. These issues may be illuminated by some preliminary results on the development of tip-link orientation, which suggest that tip links are formed from an undifferentiated population of lateral links by the differential growth of the stereocilia of the various rows (Pickles et al., 1989).

\section{Acknowledgements}

This work was supported by the Medical Research Council of the UK, the Deutsche Forschungsgemeinschaft within the programme of the SFB 204 'Gehör', the Commission of the European Community, and the Hearing and Spech Trust. The skilled technical assistance of L.M. Tompkins and T.L. Hayward is gratefully acknowledged.

\section{References}

Bagger-Sjöbäck, D. and Takumida, M. (1988) Geometrical array of the vestibular sensory hair bundle. Acta Otolaryngol. 106, 393-403.

Comis, S.D., Pickles, J.O. and Osborne, M.P. (1985) Osmium tetroxide postfixation in relation to the cross linkage and spatial organization of stereocilia in the guinea-pig cochlea. J. Neurocytol. 14, 113-130.

Comis, S.D., Pickles, J.O., Osbornc, M.P. and Pepper, C.B. (1989) Tip-link organization in anomalously-oriented hair cells of the guinea pig cochlea. Hear. Res. 40, (in press).

Csukas, S.R., Rosenquist, T.H. and Mulroy, M.J. (1987) Connections between stereocilia in auditory hair cells of the alligator lizard. Hear. Res. 30, 147-156.

Fischer, F.P., Köppl, C. and Manley, G.A. (1988) The basilar papilla of the barn owl Tyto alba: a quantitative morphological SEM analysis. Hear. Res. 34, 87-102. 
Flock, A. (1965) Electron micruscopic and electrophysiological studies on the lateral line canal organ. Acta Otolaryngol. Suppl. 199, 1-90.

Gleich, O. and Manley, G.A. (1988) Quantitative morphological analysis of the sensory epithelium of the starling and pigeon basilar papilla. Hear. Res. 34, 69-85.

Hudspeth, A.J. and Corey, D.P. (1977) Sensitivity, polarity, and conductance change in the response of vertebrate hair cells to controlled mechanical stimuli. Proc. Natl. Acad. Sci. USA. 74, 2407-2411.

Jeffries, D.J.R., Pickles, J.O., Osborne, M.P., Rhys-Evans, P.H. and Comis, S.D. (1986) Crosslinks between stereocilia in hair cells of the human and guinea pig vestibular labyrinth. J. Laryngol. Otol. 100, 1367-1374.

Little, K.F. and Neugebauer, D.Ch. (1985) Interconnections between the stereovilli of the fish inner ear. II. Systematic investigation of saccular hair bundles from Rutilus rutilus (Teleostei). Cell Tissue Res. 242, 427-432.

Lowenstein, O. and Wersäll, J. (1959) A functional interpretation of the electron-microscopic structure of sensory hairs in the cristae of the elasmobranch Raja clavata in terms of directional sensitivity. Nature 184, 1807-1808.

Manley, G.A. (1989) Peripheral Hearing Mechanisms in Reptiles and Birds. Springer, Berlin (in press).

Miller, M.R. (1974) Scanning electron microscope studies of some skink papillae basilares. Cell Tissue Res. 150, 125-141.

Miller, M.R. (1978) Further scanning electron microscope studies of lizard auditory papillae. J. Morphol. 156, 381-418.

Osborne, M.P., Comis, S.D. and Pickles, J.O. (1984) Morphology and cross-linkage of stereocilia in the guinea pig labyrinth examined without the use of osmium as a fixative. Cell Tissue Res. 237, 43-48.

Pickles, J.O. (1985) Recent advances in cochlear physiology. Prog. Neurobiol. 24, 1-42.

Pickles, J.O. (1988) An Introduction to the Physiology of Hearing, 2nd edition. Academic Press, London.

Pickles, J.O., Comis, S.D. and Osborne, M.P. (1984) Cross-links between stereocilia in the guinea pig organ of Corti, and their possible relation to sensory transduction. Hear. Res. 15, 103-112.

Pickles, J.O., Brix, J., Gleich, O., Köppl, C., Manley, G.A., Osborne, M.P. and Comis, S.D. (1988a) The fine structure and organization of tip links on hair cell stereovilli. In: $H$. Duifhuis, J.W. Horst and H.P. Wit (Eds.), Basic Issues In Hearing, Academic Press, London, pp. 56-63.

Pickles, J.O., Osborne, M.P., Comis, S.D., Köppl, C., Gleich, O., Brix, J. and Manley, G.A. (1988b) Tip-link organization in relation to the structure and orientation of stereovillar bundles. In: J.P. Wilson and D.T. Kemp (Eds.) Cochlear Mechanisms, Plenum, New York, pp. 37-46.

Pickles, J.O., Brix, J. and Gleich, O. (1989) The search for the morphological basis of mechano-transduction in cochlear hair cells. In: L.M. Aitkin and M.J. Rowe (Eds.), Information Processing in the Mammalian Auditory and Tactile Systems, Alan R. Liss, New York, (in press).

Rhys-Evans, P.H., Comis, S.D., Osborne, M.P., Pickles, J.O. and Jeffries, D.J.R. (1985) Cross-links between stereocilia in the human organ of Corti. J. Laryngol. Otol. 99, 11-19.

Tilney, L.G., Tilney, M.S., Saunders, J.C. and DeRosier, D.J. (1986) Actin filaments, stereocilia, and hair cells of the bird cochlea. III. The development and differentiation of hair cells and stereocilia. Dev. Biol. 116, 100-118.

Tilney, M.S., Tilney, L.G. and DeRosier, D.J. (1987) The distribution of hair cell bundle lengths and orientations suggests an unexpected pattern of hair cell stimulation in the chick cochlea. Hear. Res. 25, 141-151.

Tilney, L.G., Tilney, M.S. and Cotanche, D.A. (1988a) New observations on the stereocilia of hair cells of the chick cochlea. Hear. Res. 37, 71-82.

Tilney, L.G., Tilney, M.S. and Cotanche, D.A. (1988b) Actin filaments, stereocilia and hair cells of the bird cochlea. V. How the staircase pattern of stereociliary lengths is generated. J. Cell Biol. 106, 355-365.

Wever. E.G. (1978) The Reptile Ear. Princeton University Press, Princeton. 